\title{
International symposium of junior neuroscience investigators (ISJNI19) UPM, 25-27 April, 2019 (Part-VI)
}

Neuroprotective effects of Lepidium sativum $L$. aqueous extract's on Wistar rat memory

Soumia ED*, Samira Boulbaroud, Latifa DIdou, Ahmed Ahami and Fatima-Zahra Azzaoui

Unit of Behavioral and Cognitive Neuroscience and Applied Nutrition, Laboratory of Nutrition, Health and Environment, Department of Biology, Faculty of Science, Kenitra, Morocco

The aim of our study is to investigate the effect of Lepidium sativum L.on Memory and on Acetylcholinesterase (AChE) activity in different brain structures, as well as the histology of brain among cadmiumexposed rats.

21 female rats $(155.95 \pm 3.99)$ g, 3 months old were divided into three groups $(n=7): 1)$ Control group $(\mathrm{T})$ : received a distilled water orally, 2) Treated group (C): received oral administration dose of CdCl2 at $10 \mathrm{mg} / \mathrm{kg}, 3)$ Treated group (C + LS): received CdCl2 $(10 \mathrm{mg} / \mathrm{kg})$ and the aqueous extract of Lepidium sativum L. (20mg/kg) orally.The Novel Object Recognition Memory Test is used to evaluate the short and long term recognition memory. The measure of AchE activity is realized by Ellman's method.

The obtained results showed that subchronic $\mathrm{CdCl} 2$ intoxication at a dose of $10 \mathrm{mg} / \mathrm{kg}$ causeda neurobehavioral impairments including:

A significant decrease in the index of recognition of short-term memory and long-term memory $(p<0.05)$ compared to the control group. In addition, this index increase in the group $(\mathrm{C}+\mathrm{LS})$ compared to the group intoxicated by $\mathrm{CdCl} 2$. However, this increase remains less important compared to the control group.

A highly significant increase in the enzymatic activity of acetylcholinesterase in hippocampus $(p<0.001)$, cerebellum $(p<0.001)$, and cortex $(\mathrm{p}<0.001)$.

The administration of oral $\mathrm{CdCl} 2$ at a dose of $10 \mathrm{mg} / \mathrm{kg}$ caused memory impairments. However, the co-administration of $\mathrm{CdCl} 2$ and the aqueous extract of Lepidium sativum $L$ showed a neuroprotective action against cadmium-induced memory disorders.

Key words: cadmium, lepidium sativum l, wistar rats, memory, acetylcholinesteras

Effect of brain tumors on the temporal and spatial orientation among the Moroccan population

Maria legmouz ${ }^{\star}$ Azzaoui FZ, Boulbaroud S and Ahami AOT

Universite Ibn Tofail, Kenitra

Aim: The aim of our study is to investigate the possible effect of brain tumors on the temporal and spatial orientation among the Moroccan population . the study is realized in Hospital of Specialties, IBN SINA Rabat.
Methods: 75 patients, aged between 6 and 75 years old, (35 women and $40 \mathrm{men}$ ), with or without medical history, hospitalized for a brain tumor. After the diagnosis, temporal and spatial orientation are evaluated by the BENTON test.

Results: The obtained results showed that $25.33 \%$ of studied population suffer from a temporal and spatial disorientation, (14.67\% are women, $10.66 \%$ are men) against $74.67 \%$ that are well directed in time and space.

Conclusion: This preliminary results found that temporal and spatial disorientation are present in brains tumors patients. Deeper investigations are needed to study the mechanism and factors involved in this impairment.

Key words: disorientation, time, space, test benton

Controled hypothermia in term newborn after perinatal asphyxia

\section{Abdeljalil Zoubadi and Fatiha Bennaoui}

Mohammed VI University Hospital (CHU), Marrakech, Morocco

Anoxo-ischemic encephalopathy (AIE) of the full-term newborn due to perinatal asphyxia is an important cause of death $(23 \%$ of the 4 million annual neonatal deaths) and neuro-developmental sequelae ( $20 \%$ of cases of cerebral palsy of the child at term.). It occurs in 2 newborns per 1000 live births. Animal and then human studies have shown that a reduction of the central temperature by 3 to $4 \mathrm{C}^{\circ}$ could improve the fate of these newborns by exerting a neuroprotective effect. The goal of controlled hypothermia is to improve the long-term outcome of neonates with perinatal EIA.

Key words: anoxo-ischemic encephalopathy, neonatal deaths, hypothermia

The effects of chronic stress on behavior and cognition in mice

Nouhaila Chaoui*, Rachid Hnini, Mohamed Najimi and Fatiha Chigr

Biological Engineering Laboratory, Faculty of Sciences and Technologies, Sultan MoulaySlimane University, BeniMellal, Morocco

Stress has become a risk factor in the development of many diseases, it affects many functions and systems as well as the organs that control them. The nervous system remains one of the targets of choice of the stress causing in turn negative consequences and which can moreover be harmful, depending on the paradigm of stress used and its duration. The aim of our study is to evaluate the effects of a chronic variable stress (CVS) model on behavior in mice, mainly food intake, anxiety

Received: May 24, 2019; Accepted: June 14, 2019; Published: June 18, 2019 
and memory. For this, four groups were used,: G1: control, G2: group under stress, G3: control group sucrose and G4: group CVS + sucrose. After habituation, G2 and G4 mice were exposed to CVS. To evaluate memory, a Y-labyrinth test and an object recognition test were used. For anxiety, elevated plus maze, marble burying, light dark preference and open field test were used. The results show that CVS has no effect on changes in body weight and dietary intake. However, sucrose intake increased during stress in G4 mice. On the other hand, we observed a decrease in memory in stressed G2 and G4 mice. Only G2 animals have developed anxiety. In summary, CVS alters cognitive functions, including memory and elicits anxious behavior. Of note, the antioxidant status has also been shown to be altered in brain which could explain the behavioral alterations. Increasing sucrose intake may have a protective effect against stress because G4 animals have not developed anxiety compared to those in group G2.

Key words: CVS: chronic variable stress

\section{Bibliographic synthesis on wilson's disease neurological disorder}

Kabdy $H^{1}$, Lafhal $K^{1}$, Sabir ES ${ }^{1}$, Hammoud $M^{1}$, Erragbaoui $N^{1}$, EL Khiat $\mathrm{AB}^{3}$, Fdil $\mathbf{N}^{1,2}$, Gamrani $\mathrm{H}^{3}$

${ }^{1}$ Laboratory of Biochemistry Faculty of Medicine and Pharmacy, Marrakech, Cadi Ayad University, Morocco

${ }^{2}$ Biochemistry Research Team, Cadi Ayad University, Marrakech, Morocco

${ }^{3}$ Cadi Ayyad University, faculty of sciences Semlalia, Neurosciences, Pharmacology and Environment Unit, Marrakesh, Morocco

Wilson's Disease (WD) is a disruption of copper metabolism caused by a mutation of the ATP7B gene carrying copper. The disease is autosomal recessive, it is due to copper overload, mainly in the liver and brain which results in liver and neurological symptoms following copper toxicity. the diagnosis is mainly based on clinical, biochemical and ophthalmological parameters . A major diagnostic advance has been based on the introduction of direct dosing of toxic free copper, called exchangeable copper. The ratio corresponding to the ratio (copper exchangeable / total copper) allows a diagnosis of MW if it is> $18.5 \%$.

The symptoms of (WD) are expressed by chronic liver disorders that develop into fulminant hepatitis. Subsequently, neurological disorders develop, resulting in abnormal movements with resting tremor and rigidity similar to the symptoms noted in Parkinson's disease, severe phenotypes, depression, dementia, and personality have been noted in patients.

Following the biochemical confirmation diagnosis, a treatment is administered to the patient to prevent and reverse many manifestations of this disorder. Currently the treatment of (WD) is based on the lifetime of the copper chelators (D-Penicillamine [DP] or Trolovol ${ }^{\circ}$, and Triethylenetetramine or Trientine ${ }^{\oplus}$ ) which induce a urinary elimination of copper, or the of zinc salts (Wilzin ${ }^{\circledR}$ zinc acetate or zinc sulphate which inhibit the intestinal absorption of copper, Recently, tetrathiomolybdate is also marketed for the same purpose but the latter does not yet have a license placing on the market (AMM).

The undesirable and serious effects of these treatments in patients, deterioration neurological effects, immunological, hematological, digestive, dermal and other complications (7), call for research to develop other more effective alternatives, such as than active ingredients from plants.
Key words: wilson's disease, liver disorder, neurological disorder

\section{Electrophysiological and sensorimotor injuries in lead intoxicated rats are restored by curcumin I}

\section{Hind Benammi*, Erazi H EL Hiba O, Vinay L, Bras H, Viemari J and Gamrani $H$}

Neuroscience, Pharmacology and Environment Unit, faculty of Sciences Semlalia, Cadi Ayyad University, Marrakesh, Morocco

Lead $(\mathrm{Pb})$ is a heavy metal with various adverse effects on human health including mental health (Liu et al., 2017). Is known to cause several damages in the central and peripheral nervous system, which could be manifested by several neurophysiological and behavioral outcomes (Tchounwou et al., 2012). The present study was focused on: (i). the evaluation of the effect of prenatal exposure of rats to lead $(3 \mathrm{~g} / \mathrm{l})$ from neonatal to adult age on the motor/sensory performances, excitability of spinal cord during development. ii). characterization of the neuroprotective potentials of exposure to Curcumin I (175mg / $\mathrm{kg} \mathrm{BW}$ ) upon $\mathrm{Pb}$ induced neurotoxicity by means of a behavioral and electrophysiological approaches. Using electrophysiological trial, grasping, cliff avoidance tests and Catwalk dispositive we evaluated, respectively, the impairment of the excitability of spinal neuronal networks implicated in the control of locomotion and the sensorimotor functions in neonatal and young rats exposed prenatally to lead. Our finding showed primarily, an increase in spontaneous and fictive spinal activities in newborn rats at age P1 and P2, secondly, an impairment of locomotor gait from puppy age and until young age. These abnormalities were strongly attenuated by Curcumin I co-administration.

The present study showed a potent toxic effect of chronic $\mathrm{Pb}$ exposures at the CNS during development stage. Otherwise, we noted a powerful neuroprotective effect of Curcumin I against $\mathrm{Pb}$ neurotoxicity which may qualify this drug as multipotentneuroprotective agent preventing CNS neurointoxication induced by heavy metals including $\mathrm{Pb}$.

Key words: $P b$, Curcmin I, Locomotor activity, Spontaneous activity, sensorimotor functions, Catwalk, Rats

Modulatory potential of curcumin I against lead exposure induced anxiety in the brain rat: Involvement of serotonin

\section{Hind Benammi, EL hiba O, Romane A and Gamrani $\mathrm{H}$}

\section{Neuroscience, Pharmacology and Environment Team}

Anxiety is one of the most common mental disorders sharing extreme or pathological anxiety states as the primary disturbance in mood or emotional tone, with increased fear and exaggerated acute stress responses (Ninan, 2001). Medicinal plants are very variable, but some of them are used as a spice such as curcumin (Curcuma longa). Curcumin I shows a wide range of pharmacological potentialities, however, little is known about its anxiolytic properties (Arora et al., 2011, Bachetti et al., 2012). The aim of our study was to assess the anti-anxiety potential of curcumin extract against experimental lead induced-anxiety in rats. Experiments were carried out on male Wistar rats intoxicated acutely with an intraperitoneal injection of $\mathrm{Pb}(25 \mathrm{mg} /$ $\mathrm{kg} \mathrm{B.W.)} \mathrm{and/or} \mathrm{concomitantly} \mathrm{with} \mathrm{administration} \mathrm{of} \mathrm{curcumin} \mathrm{I} \mathrm{(30}$ $\mathrm{mg} / \mathrm{kg}$ B.W.) for 3 days. Using immunohistochemistry and anxiety assessment tests (dark light box and elevated plus maze), we evaluated, respectively, the expression of serotonin $(5 \mathrm{HT})$ in the dorsal raphe 
nucleus (DRN) and the anxiety state in our animals. Our results showed, for the first time, a noticeable anxiolytic effect of curcumin I against lead induced anxiety in rats and this may possibly result from modulation of central neuronal monoaminergic neurotransmission, especially serotonin, which has shown a significant reduction of the immunoreactivity within the DRN.

Key words: anxiety, curcumin I, dorsal raphe nucleus, immunohistochemistry, lead intoxication, rat, serotonin

Curcumin I restores the impairment of lead in the subcommissural organ (SCO) responsiveness: Implication of Reissner's fiber and serotonin system

\section{Hind Benammi*, EL Hiba O and Gamrani $H$}

Laboratoire des Neurosciences, Pharmacologie et Environnement, faculté des sciences Semlalia, UCAM, Marrakech, Morocco

Substantial evidence supports the neurochemical vulnerability to lead $(\mathrm{Pb})$ as one of the most potent neurotoxic heavy metals (Metwally et al., 2015). In the present study, we aimed to assess: (i) The subcommissural organ (SCO) responsiveness as a secretory circumventricular organ to chronic and acute $\mathrm{Pb}$ intoxication together with its serotoninergic innervation. (ii) The possible restorative effect of curcumin I against $\mathrm{Pb}$ intoxication under the same pathological conditions. We used immunohistochemistry with antibodies against Reissner's fiber and serotonin [5-hydroxytryptophan (5-HT)] in Wistar rats following chronic as well as acute $\mathrm{Pb}$ administration, respectively, at $25 \mathrm{mg} / \mathrm{kg}$ intraperitoneally for 3 days and $0.3 \%$ in drinking water from the intrauterine stage until 2 months of adult age. Our data showed a significant decrease in Reissner's fiber material immunoreactivity concomitant with an overall increased 5-HT innervation of the SCO and the ventricular borders. Coadministration of curcumin I $(50 \mathrm{mg} / \mathrm{kg}$ body weight) restores this impairment by reversing the effect of chronic and acute $\mathrm{Pb}$ on the secretory activity and the 5-HTergic innervation of the SCO. The investigation showed, on the one hand, the involvement of the SCO in the response to heavy metals, especially $\mathrm{Pb}$, and on the other, the beneficial corrector role of curcumin I. As a part of the circumventricular organ, known as a privileged area of brain-blood exchanges, the SCO may play a key role in the mechanism of brain defense against heavy metal neurotoxicity in rats.

Key words: $P b$, curcmin i, subcommissural organ, immunohistochemistry, reissner's fiber, serotonin, rat

Copyright: (C2019. This is an open-access article distributed under the terms of the Creative Commons Attribution License, which permits unrestricted use, distribution, and reproduction in any medium, provided the original author and source are credited. 